\title{
The influence of anaesthesia on intraoperative neuromonitoring changes in high-risk spinal surgery
}

\author{
Nathan P. Royan, Nancy Lu, Pirjo Manninen, Lakshmikumar Venkatraghavan
}

\begin{abstract}
Background: The use of intraoperative neuromonitoring is a well-established method of detecting neurologic injuries during spine surgery.Anaesthesia, especially inhalational agents, influence motor evoked potential (MEP) monitoring.The aim of our study was to compare the effect of balanced anaesthesia (BA) (intravenous plus inhalational anaesthesia) and total intravenous anaesthesia (TIVA) on the incidence of intraoperative neuromonitoring changes, interventions performed and neurological outcomes of patients following high-risk spinal surgery. Methods: After Research and Ethics Board approval, a retrospective review of 155 patients who underwent spinal surgery with MEP was performed. Data were collected on changes in MEP and/or somatosensory evoked potential, interventions performed and neurological outcomes. Patients were divided into BA and TIVA groups and data were analysed. Results: A total of 152 patients were eligible for the study (mean age $54 \pm$ 17, male: female 45:55). A BA technique was used in $62 \%$ and TIVA in $38 \%$. Desflurane ( $<0.5$ minimum alveolar concentration [MAC]) was used in $85 \%$ BA cases. Intraoperative neuromonitoring changes occurred in $11.8 \%$ (I8/I52) of cases. There was no statistical difference in the incidence of monitoring changes between BA (78\%) and TIVA (22\%) groups $(P=0.197)$. Anaesthetic or surgical interventions were performed in 12 patients, with a resolution of changes in $50 \%(P=0.455)$. All 5 patients with persistent MEP changes had worsening of existing neurological deficits postoperatively; 8 had transient MEP changes, and 2 experienced worsening of existing neurological deficits. Conclusions: We found that intraoperative neurophysiological monitoring can be performed with both BA (MAC <0.5) andTIVA in high-risk spinal surgery with no statistical difference in the incidence of intraoperative monitoring changes.
\end{abstract}

Key words: Balanced anaesthesia, inhalational anaesthesia, intravenous anaesthesia, motor evoked potentials, somatosensory evoked potentials

\section{INTRODUCTION}

Intraoperative neurophysiological monitoring is a well-established, safe and sensitive tool for detecting

Department of Anaesthesia, Toronto Western Hospital, University Health Network, University of Toronto, Toronto, Canada

Address for correspondence:

Dr. Lakshmikumar Venkatraghavan, Department of Anaesthesia, Toronto Western Hospital, 399 Bathurst Street, Toronto, Ontario, M5T2S8, Canada.

E-mail: lashmi.venkatraghavan@uhn.ca

\begin{tabular}{|l|l|}
\hline \multicolumn{2}{|c|}{ Access this article online } \\
\hline Quick Response Code: & Website: \\
\hline & www.jnaccjournal.org \\
\cline { 2 - 2 } & \\
\hline
\end{tabular}

intraoperative injuries during spine surgery. $\cdot^{[1-3]}$ Motor and somatosensory evoked potentials (SSEP) are monitored throughout the procedure, and any significant change from baseline is noted as this may indicate a compromise of the monitored pathway.

Anaesthesia, especially inhalational agents ${ }^{[4,5]}$ and muscle relaxants, ${ }^{[6]}$ are confounders for motor evoked potential (MEP) monitoring as they have deleterious effects on the amplitude of the waveform signal. ${ }^{[7]}$ Total intravenous anaesthesia (TIVA) with no

This is an open access article distributed under the terms of the Creative Commons Attribution-NonCommercial-ShareAlike 3.0 License, which allows others to remix, tweak, and build upon the work non-commercially, as long as the author is credited and the new creations are licensed under the identical terms.

For reprints contact: reprints@medknow.com

How to cite this article: Royan NP, Lu N, Manninen P, Venkatraghavan $\mathrm{L}$. The influence of anaesthesia on intraoperative neuromonitoring changes in high-risk spinal surgery. J Neuroanaesthesiol Crit Care 2017;4:159-66. 
intraoperative muscle relaxants following intubation has been suggested as the preferred anaesthetic technique for these surgeries. ${ }^{[8]}$ However, there has been a growing body of evidence on the ability to perform intraoperative MEP monitoring with the use of either inhalational anaesthesia alone ${ }^{[9]}$ or balanced anaesthesia (BA) (intravenous [IV] anaesthesia and inhalational anaesthesia). ${ }^{[10]}$ The potential benefits include decreased patient movement, increased haemodynamic stability and faster wake-up times. ${ }^{[11]}$ The possible difficulties with the addition of inhalational agents may be the decrease in the amplitude of the MEP waveform making monitoring less accurate and an increased likelihood of false positive results. ${ }^{[12]}$ There is limited information on whether a balanced technique of anaesthesia leads to increased intraoperative neuromonitoring changes. There is also a paucity of information on the intraoperative anaesthetic and surgical interventions performed on notification of an intraoperative neuromonitoring change. The purpose of this study was to compare the effect of BA (IV combined with inhalational anaesthesia) versus TIVA on the incidence of intraoperative MEP and/or SSEP changes during high-risk spinal surgery, the surgical and anaesthetic interventions performed in response to change, and the neurological outcome of patients.

\section{METHODS}

\section{Study design}

Approval was obtained from the Research and Ethics Board of our institution for the retrospective review of medical records. No patient consent was deemed necessary. We conducted a retrospective study of 155 patients between November 2011 and March 2014 who underwent spinal surgery at our institution requiring MEP monitoring as determined by the operating surgeon.

\section{Data source collection}

The electronic patient medical records were reviewed for all the identified patients. The data collected included patient demographics, pre-operative neurological function, anaesthesia information (type, haemodynamics, physiological variables and all interventions), surgical data (procedure, blood loss, complications) and details of neuromonitoring (monitoring techniques and events, all changes) post-operative neurological outcomes and any complications.

\section{Methods}

\section{Perioperative anaesthesia care}

All patients had general anaesthesia with full standard monitoring (electrocardiogram, non-invasive blood pressure, oxygen saturation, end-tidal carbon dioxide and volatile concentration). Invasive arterial and/or central venous pressure monitoring was sited at the discretion of the anaesthesiologist.

Induction of general anaesthesia was with fentanyl $2-3 \mathrm{mcg} / \mathrm{kg}$, propofol $2-3 \mathrm{mg} / \mathrm{kg}$ and rocuronium $0.6 \mathrm{mg} / \mathrm{kg}$ for intubation of the trachea with an intubation technique selected by the anaesthesiologist. Inhalational agents were used until the patients were placed in the operative position selected by the surgeons. The anaesthetic was then changed to TIVA (propofol $75-160 \mathrm{mcg} / \mathrm{kg} / \mathrm{min}$ and remifentanil $0.03-0.15 \mathrm{mcg} / \mathrm{kg} / \mathrm{min}$ ) infusions) with the optional addition of a background inhalational anaesthetic, either sevoflurane or desflurane (minimum alveolar concentration $[\mathrm{MAC}]<0.5)$. Anaesthetic regimen was titrated to Entropy (GE Healthcare, Finland) reading of 40-60. No further muscle relaxation was administered unless requested by the surgeon during surgical exposure. The patients were classified into two groups, BA or TIVA, based on the anaesthetic management of the patient during the major part of the surgical procedure where MEP and SSEP monitoring was performed.

During maintenance anaesthesia, the primary aim was for physiological stability with the maintenance of mean arterial pressure (MAP) $>80 \mathrm{mmHg}$, temperature $>35^{\circ} \mathrm{C}$, oxygen saturation $>95 \%$, end tidal carbon dioxide $<35 \mathrm{mmHg}$ and haemoglobin $>90 \mathrm{~g} / \mathrm{dl}$. Patients received additional analgesia with boluses of fentanyl 25-50 mcg and hydromorphone 0.2-0.4 mg depending on the surgical procedure. During the closure of the surgical wound neuromonitoring of MEP was stopped at the discretion of the surgeon and neurophysiologist. At this time, TIVA was discontinued and patients were changed to an inhalational anaesthetic technique. Antiemetics (ondansetron 4-8 mg IV) were given, and if required, neuromuscular blockade reversed with neostigmine $2.5 \mathrm{mg}$ and glycopyrrolate $0.4 \mathrm{mg}$.

In the absence of any surgical complications or other contraindications, patients were woken in the operating room with extubation of their trachea once they were fully awake and stable. Patients were transferred to the post-anaesthetic care unit once extubated or directly to the intensive care unit if they remained intubated.

\section{Neuromonitoring}

All intraoperative neuromonitoring was performed according to institutional guidelines by trained neurophysiologists. Baseline MEP and SSEP were obtained after positioning of the patient. For MEP, multi-pulse transcranial electric stimulation was achieved using either of two intraoperative machines, the Cadwell Cascade TCS-1 (Cadwell, Kennewick, Washington, USA) or the Natus Xltek Protektor 32 (Natus 
Medical Incorporated, Pleasanton, California, USA). Each machine delivered a constant voltage to evoke a compound muscle action potential (CMAP). A train of 5 pulses, applied for $0.05-\mathrm{ms}$ duration each, was delivered at an interstimulus interval of $2 \mathrm{~ms}(500 \mathrm{~Hz})$. Stimulating electrodes were inserted along the motor cortex at C3 and C4 (10-20 international system). The ground electrode was located at the sternum. Stimulation for the upper limbs started at $250 \mathrm{~V}$ and for lower limbs at $300 \mathrm{~V}$. Stimulation was then gradually increased until a reproducible MEP was elicited and then fixed as the baseline. The maximum output was limited to $500 \mathrm{~V}$. The MEP recordings were obtained with stainless steel hollow bore subdermal 12-mm electrodes inserted into various muscle groups. In the upper limb, CMAP was recorded from the deltoids, biceps, triceps and first dorsal interosseous muscles. In the lower limb, CMAP was recorded from the quadriceps, tibialis anterior, gastrocnemius and abductor hallucis. Baseline MEPs were obtained either before skin incision or once neuromuscular blockade given during intubation had sufficiently resolved.

The SSEPs were elicited from all four limbs. Subdermal electrodes were used, and in the upper limb, the ulnar nerve was stimulated at $20 \mathrm{~mA}$ and in the lower limb, the posterior tibial nerve was stimulated at $30 \mathrm{~mA}$. Pulse duration was $0.25 \mathrm{~ms}$ with a frequency of $2.79 \mathrm{~Hz}$. Recording electrodes were inserted along the sensory cortex at C3 and C4 (10-20 international system). Peripheral, subcortical and cortical responses were recorded throughout the surgical procedure.

According to institutional guidelines, the criteria for a significant MEP or SSEP change were a decrease in amplitude of the CMAP by $50 \%$. Changes were classed as transient if amplitudes improved to $>50 \%$ of baseline after an intervention or spontaneously during the procedure. They were considered to be persistent when there was an absence of improvement to $>50 \%$ of baseline.

All intraoperative interventions in response to a change in MEP and/or SSEP were divided into anaesthetic and surgical interventions. Anaesthetic interventions included induced hypertension (a further increase in MAP to $>80 \mathrm{mmHg}$ ), reduction or cessation of the inhalational agent, and blood transfusion and the surgical interventions included hardware change, administration of methylprednisolone and a wake-up test. Multiple anaesthetic and surgical interventions may have been performed for each case, and all concurrent interventions were classed as successful in the event of a reversal of the monitoring change. Patients with an intraoperative monitoring change were assessed for new or worsening neurological deficits compared to their pre-operative neurological function.

\section{Data analysis}

Data were analysed to determine the incidence of intraoperative changes in neuromonitoring and the differences between the BA and TIVA groups. In addition, the success of the anaesthetic and surgical interventions performed in response to change was analysed. Statistical analysis was performed between the two groups, BA versus TIVA, using descriptive statistics to characterise baseline clinical characteristics, anaesthesia data, pre- and post-operative neurological function. Continuous data were presented as mean and standard deviation (SD) or median with range. Chi-square, Fisher exact test and odds ratio (OR) were used to compare categorical non-parametric data as appropriate. Student's $t$-tests were utilised for parametric data. A $P<0.05$ was deemed statistically significant for all data.

\section{RESULTS}

During the study, 155 patients underwent major spinal surgery with MEP and SSEP monitoring. Of the 155 patients, 3 patients were excluded from this study $(n=1$ lower limb MEP absent at baseline, $n=2$ absent lower limb SSEP at baseline). The remaining 152 patients were eligible for the study. The mean (SD) age of patients was 53.8 (17.23) with $45 \%$ (69) of patients in the study male.

\section{Patient characteristics and anaesthesia}

The demographics, pre-operative neurological status and surgical indications are shown in Table 1. There was no difference between the two groups except for a higher incidence of pre-operative sensory neurological deficits in the TIVA group. Overall, $62 \%(n=94)$ of the patients had a BA technique and $38 \%(n=58)$ had TIVA. The intraoperative conditions during monitoring are shown in Table 2. In the BA group, desflurane was the volatile agent used in $85 \%(80 / 94)$ of cases with a MAC value of $<0.3$ in $38 \%$, MAC $0.3-0.5$ in $60 \%$ and $2 \%$ with MAC $>0.5$. Sevoflurane was administered in 14 patients, a MAC $<0.3$ in 5, MAC 0.3-0.5 in 8 and MAC $>0.5$ in 1. In the TIVA group, $93 \%$ of patients received propofol and remifentanil infusions, and the remaining 4 patients' propofol infusion with intermittent fentanyl boluses.

\section{Incidence of monitoring changes}

The incidences of monitoring changes are shown in Figure 1. A significant change in either MEP and/or SSEP monitoring was recorded in $11.8 \%(18 / 152)$ of the cases. Among the patients with a monitoring change, $78 \%(14 / 18)$ received $\mathrm{BA}$, and the remaining $22 \%$ received TIVA $(P=0.197)(\mathrm{OR}=2.36)$. In addition, there were no differences in the intraoperative physiological conditions under anaesthesia between the two groups except for lower entropy values and end tidal $\mathrm{CO}_{2}$ concentration in the TIVA group [Table 2]. Eight patients 
Table 1: Patient characteristics

\begin{tabular}{|c|c|c|c|}
\hline \multirow[t]{2}{*}{ All patients $(n=152)$} & \multicolumn{2}{|c|}{ Group } & \multirow[t]{2}{*}{$P$} \\
\hline & BA $(n=94)$ & TIVA $(n=58)$ & \\
\hline Age $^{*}-53.8 \pm 17.23$ & $52.9 \pm 17.88$ & $55.3 \pm 16.18$ & 0.251 \\
\hline \multicolumn{4}{|l|}{ Gender, $n(\%)$} \\
\hline Male:female & $44(47): 50(53)$ & $25(42): 33(57)$ & 0.656 \\
\hline Total & $69(45): 83(55)$ & & \\
\hline \multicolumn{4}{|l|}{ ASA $(\%)$} \\
\hline I-II & $18(19)$ & $11(19)$ & 0.978 \\
\hline III-IV & $76(81)$ & $47(81)$ & 0.978 \\
\hline \multicolumn{4}{|l|}{ Pre-operative neurological status (\%) } \\
\hline Sensory deficit & $47(50)$ & $39(67)$ & 0.037 \\
\hline Motor deficit & $33(35)$ & $24(41)$ & 0.438 \\
\hline Nil deficits & $30(32)$ & $14(24)$ & 0.304 \\
\hline Other (gait/balance issues, bowel/bladder/cranial nerve dysfunction) & $23(24)$ & $13(22)$ & 0.846 \\
\hline \multicolumn{4}{|l|}{ Surgical indication (\%) } \\
\hline Intradural & $21(22)$ & $15(26)$ & 0.620 \\
\hline Degenerative (myelopathy) & $17(18)$ & $16(27)$ & 0.168 \\
\hline Deformity (scoliosis) & $22(24)$ & $11(19)$ & 0.519 \\
\hline Vertebral tumour/metastases & $8(9)$ & $4(7)$ & 0.720 \\
\hline Fracture & $8(9)$ & $4(7)$ & 0.720 \\
\hline Other & $18(19)$ & $8(13)$ & 0.507 \\
\hline
\end{tabular}

*All other values $n$ (\%) except for age (mean and SD). BA=Balanced anaesthesia, TIVA=Total intravenous anaesthesia alone group, ASA=American Society of Anesthesiology, SD=Standard deviation

Table 2: Intraoperative conditions (patients with a monitoring change) $(n=18)$

\begin{tabular}{|c|c|c|c|}
\hline Intraoperative data & $\mathrm{BA}(n=14)$ & TIVA $(n=4)$ & $P$ \\
\hline Propofol infusion rate $(\mathrm{mcg} / \mathrm{kg} / \mathrm{min})$ & $106 \pm 22.97$ & $135 \pm 17.32$ & 0.017 \\
\hline $\begin{array}{l}\text { MAC } \\
\text { Desflurane }(n=11) \\
\text { Sevoflurane }(n=3)\end{array}$ & $0.32 \pm 0.13$ & - & 0.107 \\
\hline Entropy & $45.11 \pm 7.23$ & $36 \pm 4.69$ & 0.022 \\
\hline MAP & $78 \pm 13.85$ & $76.25 \pm 7.41$ & 0.407 \\
\hline $\mathrm{SaO}_{2} \%$ & $99.14 \pm 0.66$ & $99.25 \pm 0.9$ & 0.399 \\
\hline $\mathrm{ETCO}_{2}$ & $33.5 \pm 2.07$ & $31 \pm 0.82$ & 0.017 \\
\hline Temperature & $35.54 \pm 0.73$ & $35.58 \pm 0.57$ & 0.468 \\
\hline Lowest recorded $\mathrm{Hb}$ & $112.21 \pm 20.23$ & $107.75 \pm 18.08$ & 0.348 \\
\hline Intraoperative blood loss & $1157.14(200-3700)$ & $1337.50(450-3300)$ & 0.085 \\
\hline
\end{tabular}

had only MEP changes, 5 patients had only SSEP changes and 5 patients had changes in both MEP and SSEP. There was no statistical difference between the two groups with respect to MEP and SSEP changes.

\section{Intervention with monitoring change and outcome}

Out of 18 patients who had intraoperative monitoring change, $12(66 \%)$ patients (BA 10, TIVA 2) $(P=0.569)$ had anaesthesia and/or surgical interventions. The interventions used are shown in Table 3. Overall, 6 of the 12 patients (BA 4, TIVA 2$)(P=0.455)$ that received an intervention had a recovery of their monitoring changes. However, in 6 patients, the monitoring changes remained persistent despite the interventions. No intervention was documented in 6 patients, 3 of these changes were transient, and 3 were persistent. Induced hypertension (MAP >80) was attempted in 


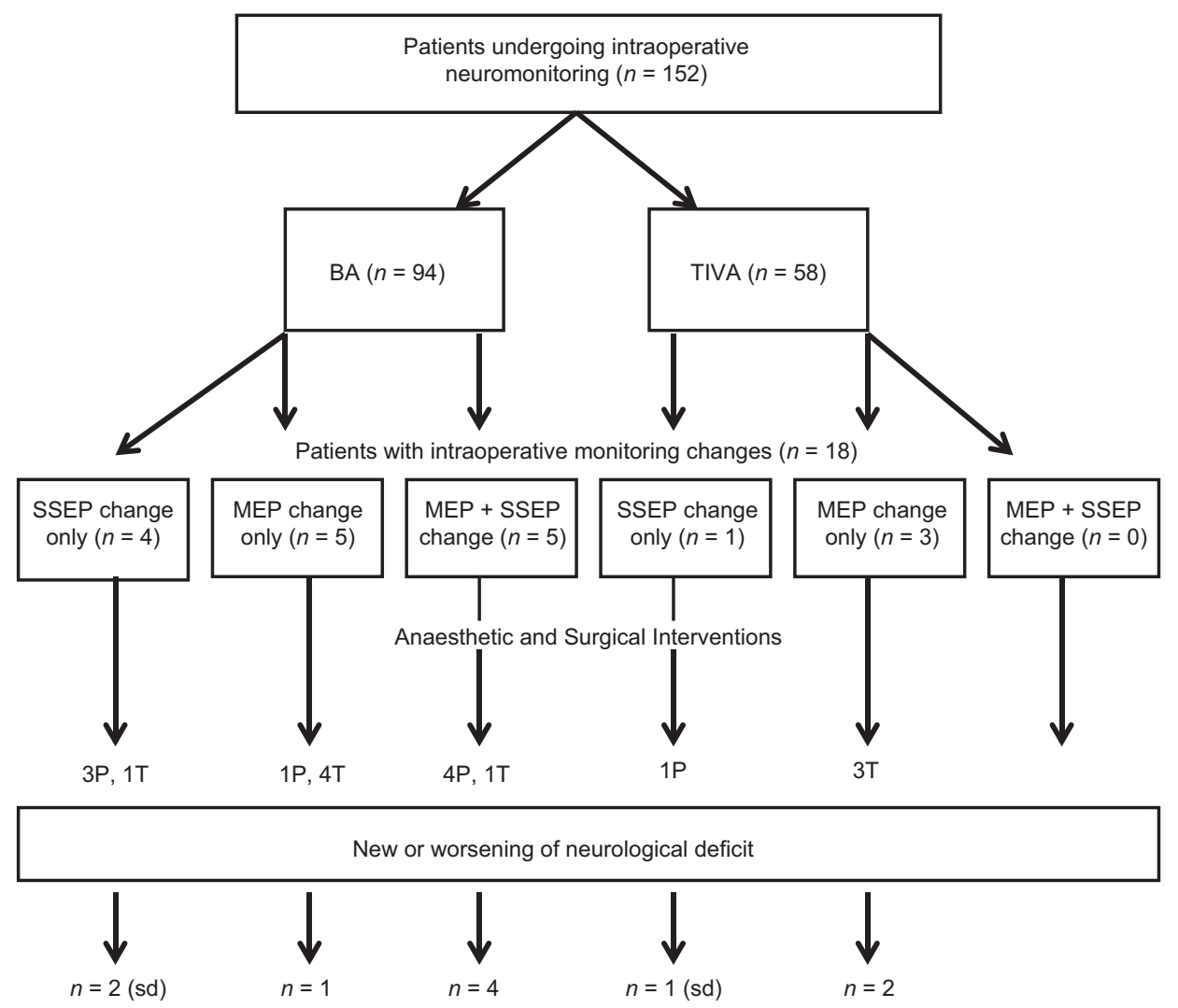

Figure 1: Flowchart for monitoring changes. $B A=$ Balanced Anaesthesia, TIVA= Total Intravenous Anaesthesia, MEP= Motor evoked potentials, SSEP $=$ Somatosensory evoked potentials, $\mathrm{P}=$ persistent monitoring change, $\mathrm{T}=$ transient monitoring change, sd= sensory deficit only

Table 3: Monitoring changes, interventions and outcomes

\begin{tabular}{|c|c|c|c|c|c|c|}
\hline Patient & $\begin{array}{c}\text { Anaesthesia } \\
\text { group }\end{array}$ & $\begin{array}{c}\text { Pre-operative } \\
\text { neurological deficit }\end{array}$ & $\begin{array}{l}\text { MEP } \\
\text { change }\end{array}$ & $\begin{array}{l}\text { SSEP } \\
\text { change }\end{array}$ & Interventions & $\begin{array}{l}\text { New or worsening } \\
\text { neurological deficit }\end{array}$ \\
\hline 1 & BA & Yes & Persistent & Persistent & $\mathrm{BP}, \mathrm{IA}, \mathrm{BT}$ & Yes \\
\hline 2 & BA & Yes & Persistent & Persistent & $\mathrm{BP}, \mathrm{HW}, \mathrm{MP}$ & Yes \\
\hline 3 & BA & Yes & Persistent & Persistent & BT & Yes \\
\hline 4 & BA & Yes & Persistent & - & $\mathrm{BP}, \mathrm{IA}$ & Yes \\
\hline 5 & BA & Yes & Transient & Transient & - & No \\
\hline 6 & BA & Yes & Persistent & Persistent & $\mathrm{BP}, \mathrm{IA}$ & Yes \\
\hline 7 & BA & No & Transient & - & BT & No \\
\hline 8 & BA & Yes & Transient & - & $\mathrm{BP}$ & No \\
\hline 9 & BA & Yes & Transient & - & IA & No \\
\hline 10 & TIVA & Yes & Transient & - & - & Yes \\
\hline 11 & BA & No & Transient & - & $\mathrm{BP}, \mathrm{IA}$ & No \\
\hline 12 & TIVA & Yes & Transient & - & HW & No \\
\hline 13 & TIVA & Yes & Transient & - & BT, HW, WU & Yes \\
\hline 14 & BA & Yes & - & Persistent & $\mathrm{BP}$ & No \\
\hline 15 & BA & No & - & Persistent & - & Yes (SD) \\
\hline 16 & TIVA & No & - & Persistent & - & Yes (SD) \\
\hline 17 & BA & Yes & - & Transient & - & No \\
\hline 18 & BA & Yes & - & Persistent & - & Yes (SD) \\
\hline
\end{tabular}

MEPs=Motor evoked potentials, SSEP=Somatosensory evoked potentials, BA=Balanced anaesthesia, TIVA=Total intravenous anaesthesia, BP=Increase in blood pressure, IA=Decrease in inhalation al anaesthesia, $\mathrm{BT}=\mathrm{Blood}$ transfusion, $\mathrm{HW}=$ Change in hardware, $\mathrm{MP}=$ Methylprednisolone, $\mathrm{WU}=\mathrm{Wake}-\mathrm{up}$ test, $\mathrm{SD}=$ Standard deviation 
7 cases and in 3 cases there was a subsequent resolution of the monitoring change. The dose of inhalational anaesthesia was reduced or ceased in 5 cases and in 2 cases monitoring changes resolved. Blood transfusion was performed in 3 cases and resulted in the resolution of the MEP change in 1 case.

Surgical intervention was attempted in 3 of the 18 cases where monitoring changes occurred. In one patient this involved a hardware change and administration of methylprednisolone and was unsuccessful in resolving the change. In the two other patients, a hardware change was implemented, and recovery of MEP changes followed. In one of these patients, additional interventions namely blood transfusion and intraoperative wake-up test were also performed, and there was the resolution of the monitoring change.

\section{Neurological status and outcome}

Preoperatively, $71 \%(n=108)$ of the patients presented with a neurological deficit and incidence was similar between the groups [Table 1]. MEP changes were recorded in 13 patients. All of the 5 patients who had persistent MEP changes had pre-operative neurological deficits that worsened postoperatively. This was in comparison to the 8 patients that had transient MEP changes where only 2 patients had a new or worsening post-operative neurological deficit $(P=0.021)$. Notably, all patients that awoke from surgery with a new or worsening neurological deficit following an intraoperative monitoring change had a pre-existing neurological deficit preoperatively [Table 3].

Isolated SSEP changes were recorded in 5 patients. These changes were persistent in 4 cases and transient in the remaining case. Pre-operative neurological deficits were present in 3 of these patients. In these patients, there were no new post-operative motor deficits, but 3 of these patients had new post-operative sensory deficits. There was no statistical relationship between SSEP changes and neurological outcome.

\section{DISCUSSION}

In our study, we found that intraoperative neurophysiological monitoring can be performed with both BA and TIVA. We found no significant difference in the incidence of intraoperative changes or the interventions performed in the event of neuromonitoring changes between the BA and TIVA groups. In total, a significant change in MEP and/or SSEP was recorded during $11.8 \%$ of all procedures, with BA being used in $78 \%$ of these cases. Interventions were performed in $66 \%$ (12) of the patients who had a monitoring change, and the intervention was successful in the recovery of the MEP change in 6 patients. Induced hypertension and decreasing the concentration of the inhalational agents were the most common anaesthesia interventions, and a hardware change was the most common surgical intervention.

The effect of anaesthetic agents on MEP monitoring is well documented with the reduction of the MEP waveform in a dose-dependent manner, ${ }^{[4]}$ which may increase the difficulty in recording changes related to nerve or spinal cord injury. To decrease the anaesthetic depression of MEP, TIVA has often been recommended as the effects of propofol may be less than inhalation agents. The use of inhalational anaesthesia for MEP monitoring has been documented to be successful with lower concentrations (MAC <0.5). ${ }^{[10]}$ A recent study compared the use of desflurane/remifentanil and propofol/remifentanil in patients with no pre-operative neurological deficits and found that monitoring of MEP was possible in all patients but in patients who had small amplitude baseline waveforms desflurane produced a greater reduction in amplitude. ${ }^{[9]}$

In previous studies in patients undergoing scoliosis surgery, the incidence of a significant intraoperative monitoring change has been reported to occur from $3.6 \%$ to $8.7 \%{ }^{[13-15]}$ We recognised an increased incidence of $11.8 \%$ for intraoperative neuromonitoring changes. In this study, we assessed patients undergoing high-risk spinal surgery, where $71 \%$ of patients had pre-operative neurological deficits. Of the patients who had a change in monitoring, 78\% (14/18) had pre-operative neurological deficits. The presence of pre-operative neurological deficits may impair the ability to effectively monitor as baseline MEP and SSEP may be affected with poor amplitude of the waveform. ${ }^{[16]}$ Notably, in previous studies performed patients often have no pre-operative deficits or information on pre-operative neurological status is limited.

We observed that more patients receiving BA experienced an MEP change more frequently but this did not achieve statistical significance as the number of patients was small. Moreover, we also postulate that the groups were unmatched due to a possible anaesthetist bias towards TIVA in patients with pre-operative sensory and motor deficits. The incidence of fewer MEP changes in the TIVA group that had significantly poorer pre-operative neurological function also raises the question of the contribution of BA to intraoperative MEP changes.

The anaesthetic interventions performed had limited success in our study compared to other studies. ${ }^{[14]}$ In the study by Samdani et al. 36 patients (5.3\%) had an MEP change, and there was a total of 47 different interventions performed. The most commonly used (in $61 \%$ of patients) was the elevation of the MAP to at least $80 \mathrm{mmHg}$. The interventions included were similar to our study and 
included a wake-up test and surgical correction. They found that the interventions were successful in $94 \%$ of patients (34/36) as compared to a success rate of $50 \%$ in our study. The Samdani study was performed in patients undergoing scoliosis surgery and there was no documentation of pre-operative neurological function. A pre-operative neurological deficit may predispose to increased risk of post-operative injury or may simply make an accurate assessment of neurological function postoperatively more challenging. The former was demonstrated in our study by the fact that all patients that awoke from surgery with new or worsening neurological deficits had a pre-operative neurological deficit. ${ }^{[2]}$ While anaesthesia interventions were not exceedingly successful in these high-risk patients, the utility of post-operative blood pressure management following monitoring changes must not be questioned due to the magnitude of the risk of spinal cord ischaemia to patients. ${ }^{[17,18]}$

The assessment of the incidence of immediate post-operative neurological deficits was defined as a new deficit or worsening of pre-operative neurological deficits. The question of true and false positive and negative findings with MEP/SSEP monitoring can difficult to define, and due to small sample size of our study group, the role of the technique of anaesthesia was difficult to interpret. ${ }^{[12]}$ False positives results may arise from many sources including anaesthesia effects especially anaesthetic fade or even technical problems. ${ }^{[19]}$ When MEP changes are transient but result in post-operative neurological deficits it is considered that these were reversible signal changes (RSCs) in the monitoring that occurred in the setting of actual intraoperative injury. ${ }^{[20]}$ We propose that some RSCs predict impending new deficits and that recovery after a surgical or anaesthetic intervention increases the likelihood of unaltered neurologic status.

\section{Study limitations}

The most important limitation in this study is the retrospective study design, and as a result, we were limited to the data recorded in the patient chart and the data captured by the neurophysiology team at the time of the operation. Second, there might be a selection bias by anaesthetists in their choice of anaesthesia in patients with poorer pre-operative neurological function. TIVA was usually preferred in these patients. Third, the implementation of multiple interventions concurrently in the setting of a monitoring change made an analysis of the outcome of anaesthetic and surgical interventions challenging. Fourth, the analysis of the neurological function of patients pre- and post-operatively was impaired by the documentation undertaken by the treating team and also the inter-rater variability of the motor grading scale utilised. ${ }^{[20]} \mathrm{A}$ more thorough composite motor grading would have allowed the availability of more information with likely better accuracy. Finally, the small sample size relative to the prevalence of intraoperative changes limits the power of the study. Hence, finding a statistically significant result when comparing BA and TIVA for their incidence of monitoring changes was unachievable. There is scope for a larger randomised controlled trial comparing BA and TIVA alone to establish whether the addition of inhalational anaesthesia in the anaesthetic regimen does lead to increased intraoperative monitoring changes.

\section{CONCLUSION}

We found that intraoperative neurophysiological monitoring can be performed with both BA (MAC <0.5) and TIVA in high-risk spinal surgery with no statistical difference in the incidence of intraoperative monitoring changes. The effect of anaesthetic agents on MEP is dose-dependent. The role of the anaesthesiologist is to be aware of monitoring changes and institute the appropriate changes in anaesthesia and treatment to ensure the best neurological outcome.

\section{Financial support and sponsorship} Nil.

\section{Conflicts of interest}

There are no conflicts of interest.

\section{REFERENCES}

1. Fehlings MG, Brodke DS, Norvell DC, Dettori JR. The evidence for intraoperative neurophysiological monitoring in spine surgery: Does it make a difference? Spine (Phila Pa 1976) 2010;35 9 Suppl: S37-46.

2. Thuet ED, Winscher JC, Padberg AM, Bridwell KH, Lenke LG, Dobbs $\mathrm{MB}$, et al. Validity and reliability of intraoperative monitoring in pediatric spinal deformity surgery: A 23-year experience of 3436 surgical cases. Spine (Phila Pa 1976) 2010;35:1880-6.

3. Kundnani VK, Zhu L, Tak H, Wong $\mathrm{H}$. Multimodal intraoperative neuromonitoring in corrective surgery for adolescent idiopathic scoliosis: Evaluation of 354 consecutive cases. Indian J Orthop 2010;44:64-72.

4. Calancie B, Klose KJ, Baier S, Green BA. Isoflurane-induced attenuation of motor evoked potentials caused by electrical motor cortex stimulation during surgery. J Neurosurg 1991;74:897-904.

5. Haghighi SS, Madsen R, Green KD, Oro JJ, Kracke GR. Suppression of motor evoked potentials by inhalation anesthetics. J Neurosurg Anesthesiol 1990;2:73-8.

6. Kim WH, Lee JJ, Lee SM, Park MN, Park SK, Seo DW, et al. Comparison of motor-evoked potentials monitoring in response to transcranial electrical stimulation in subjects undergoing neurosurgery with partial vs. no neuromuscular block. Br J Anaesth 2013;110:567-76.

7. Sekimoto K, Nishikawa K, Ishizeki J, Kubo K, Saito S, Goto F. The effects of volatile anesthetics on intraoperative monitoring of myogenic motor-evoked potentials to transcranial electrical 
stimulation and on partial neuromuscular blockade during propofol/fentanyl/nitrous oxide anesthesia in humans. J Neurosurg Anesthesiol 2006;18:106-11.

8. Chen Z. The effects of isoflurane and propofol on intraoperative neurophysiological monitoring during spinal surgery. J Clin Monit Comput 2004;18:303-8.

9. Malcharek MJ, Loeffler S, Schiefer D, Manceur MA, Sablotzki A, Gille J, et al. Transcranial motor evoked potentials during anesthesia with desflurane versus propofol - A prospective randomized trial. Clin Neurophysiol 2015;126:1825-32.

10. Chong CT, Manninen P, Sivanaser V, Subramanyam R, Lu N, Venkatraghavan L. Direct comparison of the effect of desflurane and sevoflurane on intraoperative motor-evoked potentials monitoring. J Neurosurg Anesthesiol 2014;26:306-12.

11. Martin DP, Bhalla T, Thung A, Rice J, Beebe A, Samora W, et al. A preliminary study of volatile agents or total intravenous anesthesia for neurophysiological monitoring during posterior spinal fusion in adolescents with idiopathic scoliosis. Spine (Phila Pa 1976) 2014;39:E1318-24.

12. Tamkus AA, Rice KS, Kim HL. Differential rates of false-positive findings in transcranial electric motor evoked potential monitoring when using inhalational anesthesia versus total intravenous anesthesia during spine surgeries. Spine J 2014;14:1440-6.

13. Buckwalter JA, Yaszay B, Ilgenfritz RM, Bastrom TP, Newton PO; Harms Study Group. analysis of intraoperative neuromonitoring events during spinal corrective surgery for idiopathic scoliosis. Spine Deform 2013;1:434-8.

14. Samdani AF, Bennett JT, Ames RJ, Asghar JK, Orlando G, Pahys JM, et al. Reversible intraoperative neurophysiologic monitoring alerts in patients undergoing arthrodesis for adolescent idiopathic scoliosis: What are the outcomes of surgery? J Bone Joint Surg Am 2016;98:1478-83.

15. Polly DW Jr., Rice K, Tamkus A. What is the frequency of intraoperative alerts during pediatric spinal deformity surgery using current neuromonitoring methodology? A retrospective study of 218 surgical procedures. Neurodiagn J 2016;56:17-31.

16. Kombos T, Kopetsch O, Suess O, Brock M. Does preoperative paresis influence intraoperative monitoring of the motor cortex? J Clin Neurophysiol 2003;20:129-34.

17. Vale FL, Burns J, Jackson AB, Hadley MN. Combined medical and surgical treatment after acute spinal cord injury: Results of a prospective pilot study to assess the merits of aggressive medical resuscitation and blood pressure management. J Neurosurg 1997;87:239-46.

18. Rogers WK, Todd M. Acute spinal cord injury. Best Pract Res Clin Anaesthesiol 2016;30:27-39.

19. Lyon R, Feiner J, Lieberman JA. Progressive suppression of motor evoked potentials during general anesthesia: The phenomenon of "anesthetic fade". J Neurosurg Anesthesiol 2005;17:13-9.

20. James MA. Use of the Medical Research Council muscle strength grading system in the upper extremity. J Hand Surg Am 2007;32:154-6. 